\title{
Dynamical control of correlated states in a square quantum dot
}

\author{
C. E. Creffield and G. Platero \\ Instituto de Ciencia de Materiales (CSIC), Cantoblanco, E-28049, Madrid, Spain
}

(Received 11 July 2002; published 4 December 2002)

\begin{abstract}
In the limit of low particle density, electrons confined to a quantum dot form strongly correlated states termed Wigner molecules, in which the Coulomb interaction causes the electrons to become highly localized in space. By using an effective model of Hubbard-type to describe these states, we investigate how an oscillatory electric field can drive the dynamics of a two-electron Wigner molecule held in a square quantum dot. We find that, for certain combinations of frequency and strength of the applied field, the tunneling between various charge configurations can be strongly quenched, and we relate this phenomenon to the presence of anticrossings in the Floquet quasi-energy spectrum. We further obtain simple analytic expressions for the location of these anti-crossings, which allows the effective parameters for a given quantum dot to be directly measured in experiment, and suggests the exciting possibility of using ac-fields to control the time evolution of entangled states in mesoscopic devices.
\end{abstract}

DOI: 10.1103/PhysRevB.66.235303

PACS number(s): 73.63.Kv, 42.50.Hz, 03.67.Lx

\section{INTRODUCTION}

The study of quantum coherent effects in mesoscopic systems, such as quantum dots (QDs), is a subject of great current interest, both from the theoretical point of view, and because of a growing number of possible experimental applications. One of the most notable of these is the swiftly developing field of quantum computation, in which the coherent manipulation of entangled quantum states is an essential component. Recent experimental successes in detecting Rabi oscillations in QD systems driven by ac-fields ${ }^{1}$ have spurred interest in the use of intense ac-fields to coherently manipulate the time development of electronic states. ${ }^{2}$ An exciting possibility is to make use the phenomenon of coherent destruction of tunneling (CDT), ${ }^{3}$ in which the tunneling dynamics of a quantum system become suppressed at certain parameters of the field. Tuning the driving field thus provides a simple mechanism to localize or move charge within the QD on a rapid time-scale by destroying or restoring the tunneling between regions of the device, so allowing ac-fields to be used as "electron tweezers."

In this article we study the use of ac-fields for this purpose, by investigating the time-dependent behavior of electrons confined to a two-dimensional QD with a square geometry, under the influence of a strong driving field. We use an effective model of Hubbard type to describe the system, which gives a considerable computational advantage over standard numerical approaches, and also allows us to easily include the important effects of the electron correlations produced by the Coulomb interaction. By integrating the Schrödinger equation in time, we find that, for certain values of the field, tunneling between different charge configurations within the QD can be quenched extremely well. In particular we find that tunneling processes parallel to the field can be destroyed while transverse tunneling is left unchanged, resulting in an effective decoupling between the two halves of the QD. We explain these findings by making use of the Floquet approach, ${ }^{4}$ and show that the points at which tunneling is quenched correspond to anti-crossings between Floquet quasi-energies, the locations of which can be found ac- curately using a perturbational method. This significantly clarifies how time-dependent electric fields can affect the charge distribution inside a strongly correlated QD, and how they can be used for quantum control.

At the low electron densities typically present in QDs, correlations produced by the Coulomb interaction can significantly influence the electronic structure. Such strongly correlated problems are notoriously difficult to treat, and the addition of a time-dependent field complicates the problem even further. When the mean interelectron separation exceeds a certain critical value, however, a considerable simplification occurs, as the Coulomb interaction dominates the kinetic energy and drives a transition to a quasi-crystalline arrangement which minimizes the total electrostatic energy. In analogy to the phenomenon of Wigner crystallization in bulk two-dimensional systems, such a state is termed a Wigner molecule. As the electrons in the Wigner state are sharply localized in space, the system can be naturally and efficiently discretized by placing lattice points just at these spatial locations. A many-particle basis can then be constructed by taking Slater determinants of single-particle states defined on these lattice sites, from which an effective Hamiltonian of Hubbard type can be generated to describe the low-energy dynamics of the system. ${ }^{5}$ A major advantage of this technique over standard discretization ${ }^{6}$ schemes, in which a very large number of lattice points is taken to approximate the continuum limit, is that the dimension of the effective Hamiltonian is much smaller (typically by many orders of magnitude), which permits the investigation of systems which would otherwise be prohibitively complex. This approach has proven to be extremely successful in treating a variety of static problems, including one-dimensional QDs, ${ }^{5}$ two-dimensional QDs with polygonal boundaries, ${ }^{7,8}$ and electrons confined to quantum rings. ${ }^{9}$ We further develop this method in this work by including a time-dependent electric field, and study the temporal dynamics of the system as it is driven out of equilibrium.

\section{MODEL AND METHODS}

We consider a system of two electrons confined to a square QD with a hard-wall confining potential-a simple 
(a)
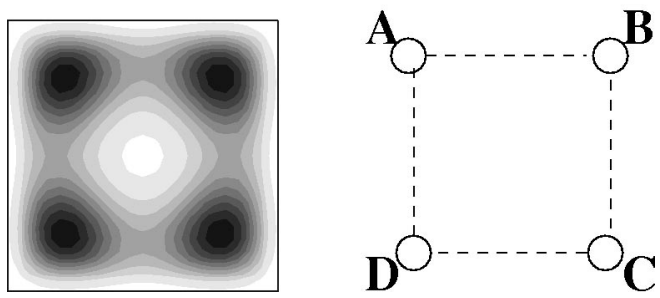

FIG. 1. (a) Ground-state charge-density for a two-electron square QD. GaAs material parameters are used, and the side-length of the QD is $800 \mathrm{~nm}$, placing it in the Wigner regime. The dark areas indicate peaks in the charge-density. (b) Lattice points used for the effective lattice-Hamiltonian.

representation of a two-dimensional semiconductor QD. Such a system can be produced by gating a two-dimensional electron gas confined at a heterojunction interface, and by placing a gate split into four quadrants over the heterostructure, ${ }^{10}$ the potentials at the corners of the QD can be individually regulated. In Fig. 1(a) we show the groundstate charge-density obtained from the exact diagonalization of a square QD, ${ }^{7}$ for device parameters placing it deep in the Wigner molecule regime. It can be seen that the chargedensity is sharply peaked at four points, located close to the vertices of the QD. This structure arises from the Coulomb interaction between the electrons, which tends to force them apart into diagonally opposite corners of the dot. As there are two such diagonal states, degenerate in energy, we can understand the form of the ground-state by considering it to be essentially a superposition of these two states (with a small admixture of higher energy states). The four points at which the peaks occur define the sites on which the effective latticeHamiltonian operates, as shown in Fig. 1(b).

We take an effective lattice-Hamiltonian of the form

$$
\begin{aligned}
H= & \sum_{\langle i, j\rangle, \sigma}\left[\tilde{t}\left(c_{i \sigma}^{\dagger} c_{j \sigma}+\text { H.c. }\right)+\widetilde{V} n_{i} n_{j}\right]+\sum_{i}\left[\widetilde{U} n_{i \uparrow} n_{i \downarrow}\right. \\
& \left.+E_{i}(t) n_{i}\right]
\end{aligned}
$$

where $c_{i \sigma}^{\dagger} / c_{i \sigma}$ are the creation/annihilation operators for an electron of spin $\sigma$ on site $i, n_{i \sigma}=c_{i \sigma}^{\dagger} c_{i \sigma}$, and $n_{i}$ is the total charge occupation of site $i$. The quantity $\tilde{t}$ denotes the hopping between adjacent sites, and throughout this work we set $\tilde{t}$ and $\hbar$ equal to one, and measure all energies in units of $\tilde{t}$. $\widetilde{V}$ represents the Coulomb repulsion between electrons occupying neighboring sites, and $\widetilde{U}$ is the standard Hubbard $U$-term, giving the energy cost for double-occupation of a site. $E_{i}(t)$ denotes the electric potential at site $i$, which in general can have a static and a time-dependent component. In experiment, static offsets can arise either from deviations of the confining potential of the QD from the ideal geometry, or by the deliberate application of gating voltages to the corners of the QD. Applying corner potentials in this way could be used to enhance the stability of the Wigner molecule state, especially if the confining potential is softer than the hard-wall potential considered here. Corner gates may

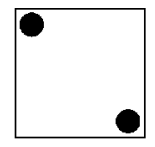

(1)

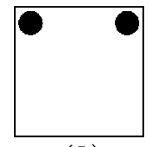

(3)

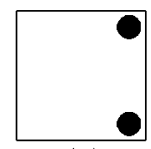

(4)

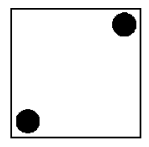

(2)

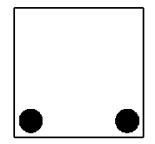

(5)

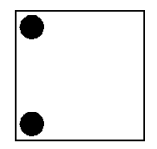

(6)
FIG. 2. Schematic representation of the two-particle basis states for the singlet subspace of the Hamiltonian. The ground state of the QD is approximately a superposition of states (1) and (2).

also be used to ensure that the multiplet of states included in this effective lattice-model is well-separated from the other excited states of the QD system, which will therefore not influence the system's dynamics. In this work, however, we do not explicitly consider the effects of static gates, and we neglect the influence of small, accidental offsets encountered in experiment as we expect them to have only minor effects, and indeed may even stabilize CDT. ${ }^{11}$ For convenience, we consider applying an ac-field aligned with the $x$-axis of the QD, which can be parametrized as

$$
E_{A}=E_{D}=\frac{E}{2} \cos \omega t, \quad E_{B}=E_{C}=-\frac{E}{2} \cos \omega t,
$$

where $A, B, C, D$ label the sites as shown in Fig. 1(b). We emphasize that although we have the specific system of a semiconductor QD in mind, the effective-Hamiltonian we are using can describe a wide range of physical systems, including $2 \times 2$ arrays of connected QDs ${ }^{12}$ and the quantum cellular automata systems studied by Lent et al., ${ }^{13}$ and our results are thus of general applicability.

We study the dynamics of the system by placing it in a certain initial state, and then integrating this state in time under the influence of the effective Hamiltonian (1), using a fourth-order Runge-Kutta method. ${ }^{14}$ During the timeevolution, typically of the order of 50 periods of the driving field, we measure physical quantities such as the particle occupation of the sites $n_{i}(t)$, and also ensure that at all times the unitarity of the wavefunction is accurately preserved. As we consider a two-electron system, its eigenstates are symmetric (antisymmetric) under particle exchange, corresponding to their singlet (triplet) symmetry. The Hamiltonian (1) contains no spin-flip terms since measurements on semiconductor QDs show that the spin-flip relaxation time is typically extremely long, ${ }^{15}$ and so the singlet and triplet subspaces are completely decoupled. Thus if the initial state possesses a definite parity, this will be retained throughout its time evolution, and we only need to include states of the same parity in the basis. We choose to use initial states with singlet symmetry, which corresponds to the symmetry of the system's ground-state. Simple state counting reveals that the singlet subspace has a dimension of ten, and can be spanned by the six states shown schematically in Fig. 2, together with the four states in which each site is doubly-occupied. 
Since the Hamiltonian (1) is periodic in time, we can use the Floquet theorem to write the solutions of the timedependent Schrödinger equation as $\psi(t)=\exp \left(-i \epsilon_{j} t\right) \phi_{j}(t)$, where $\epsilon_{j}$ is called the quasi-energy, and $\phi_{j}(t)$ is a function with the same period as the driving field, called the Floquet state. This type of expression is familiar in the context of solid-state physics, where spatial periodicity permits an analogous rewriting of the spatial wavefunction in terms of quasi-momenta and Bloch states (Bloch's theorem). The Floquet states, and their corresponding quasi-energies, can be obtained from the eigenvalue equation:

$$
\left[H(t)-i \frac{\partial}{\partial t}\right] \phi_{j}(t)=\epsilon_{j} \phi_{j}(t),
$$

which, as we show in the Appendix, is the key to obtaining an analytic expression for the quasi-energies by means of perturbation theory.

Using the Floquet states as a basis, the time-evolution of a general state driven by the periodic field may be written as

$$
\psi(t)=\sum_{j} c_{j} \mathrm{e}^{-i \epsilon_{j} t} \phi_{j}(t),
$$

which is formally analogous to the standard expansion in the eigenvectors of a time-independent Hamiltonian. Indeed, in the adiabatic limit, $T=2 \pi / \omega \rightarrow \infty$, the quasi-energies evolve to the eigenenergies, and the Floquet states to the eigenstates. An important property of expanding in Floquet states (4) is that it provides a extremely valuable separation of timescales. Although the Floquet states explicitly depend on time, they are periodic with the same period as the driving field and so just influence the dynamics on short time-scales. Consequently, the long time-scale dynamics of the system is essentially determined by just the quasi-energies, and hence evaluating the quasi-energies provides a simple and direct way of investigating the long time-scale behavior of the system. In particular, when two quasi-energies are close to degenerate, the time-scale for tunneling between the states becomes extremely long, producing the phenomenon of CDT. $^{3,14}$

As the ac-field is aligned with the $x$-axis of the QD, the Hamiltonian (1) is invariant under the composite parity operation $x \rightarrow-x ; t \rightarrow t+T / 2$. As a result the Floquet states can also be classified into parity classes, depending whether they are odd or even under this parity operation. Quasi-energies belonging to different parity classes may cross, but if they belong to the same class the von Neumann-Wigner theorem forbids this, and consequently at close approaches they form anti-crossings instead. Identifying the presence of crossings and anti-crossings in the quasi-energy spectrum provides a necessary (though not sufficient) condition for CDT to occur.

The locations of these close approaches between quasienergies may be found by an analytic approach used by Holthaus ${ }^{16}$ to treat noninteracting electrons, and later generalized in Ref. 14 to include interactions. In this method the quasi-energies are obtained by first solving the Floquet equation (3) in the absence of the tunneling terms, and then performing perturbation theory with the tunneling terms acting as the "perturbation." A full description of the method is

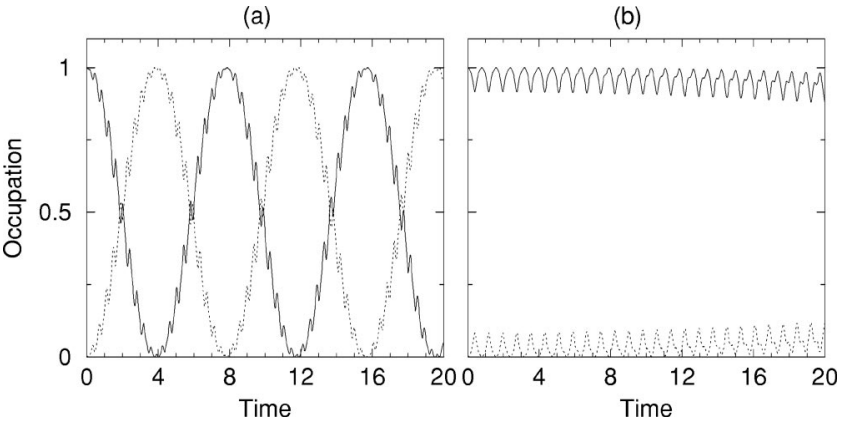

FIG. 3. Time development of the noninteracting system for $\omega$ $=8$ : (a) electric potential, $E=30.0$ (b) $E=19.24$. Solid line indicates the occupation of sites $\mathrm{A}$ and $\mathrm{D}$, the dotted line the occupation of sites $\mathrm{B}$ and $\mathrm{C}$.

given in the Appendix, and it has been shown to work extremely well when the tunneling terms are small in comparison to the other energy-scales of the problem.

A convenient numerical method to obtain the quasienergies and Floquet states is to diagonalize the unitary timeevolution operator for one period of the driving field $U(t$ $+T, t)$. It may be easily shown that the eigenvectors of this operator are equal to the Floquet states, and its eigenvalues are related to the quasi-energies via $\lambda_{j}=\exp \left[-i \epsilon_{j} T\right]$. This method is particularly well-suited to our approach, as $U(T, 0)$ can be obtained by propagating the unit matrix in time over one period of the field, using the Runge-Kutta method described earlier.

\section{RESULTS}

\section{A. Noninteracting case}

We begin our investigation by first considering the simplest case, that of noninteracting electrons $(\widetilde{V}=0, \widetilde{U}=0)$. In Fig. 3 we show the time-evolution of the system for two strengths of electric field at a frequency of $\omega=8$, in each case using state (6) (see Fig. 2) as the initial state. A consequence of using this initial state is that as the ac-field is aligned with the $x$-axis of the QD, it does not break the reflection symmetry between the upper and lower halves of the QD, and thus throughout the time evolution $n_{A}=n_{D}$, and $n_{B}=n_{C}$. For the first case, when the electric potential $E$ $=30.0$, it can be seen that the occupation of sites cycles between zero and one, as the two electrons perform spatial Rabi oscillations between the left side of the system and the right side. The picture in the second case, however, for $E$ $=19.24$, is radically different, with the occupation of sites A and D varying little from its initial value of one, and sites B and $\mathrm{C}$ remaining almost empty throughout the time evolution. It thus appears that at this second value of $E$ the tunneling between the left and right sides has been considerably suppressed.

To confirm that CDT is occurring, we present in Fig. 4 a comparison of the amplitude of the oscillations of the occupation number of site A with the quasi-energy spectrum of the system as a function of the electric potential $E$. It can be clearly seen that for most field strengths the charge oscilla- 


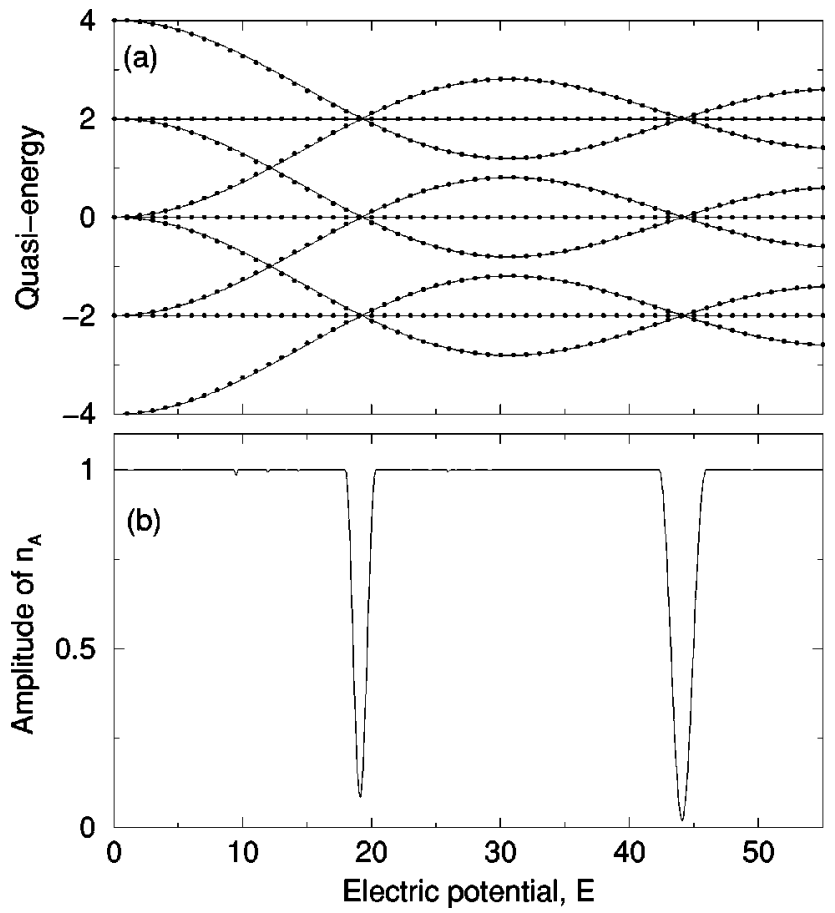

FIG. 4. (a) Quasi-energies of the noninteracting system for $\omega$ $=8$ : circles $=$ exact results, lines $=$ perturbative solution. (b) Amplitude of oscillation of the occupation of site A.

tion has an amplitude of approximately one, except at sharply defined minima where it is heavily quenched. The positions of these minima correspond precisely to the locations of exact crossings of the quasi-energies. Using the perturbative method described in the Appendix reveals that the quasi-energies fall into three bands. The central band has quasi-energies of $\epsilon_{ \pm}= \pm 2 J_{0}(E / \omega)$ (where $J_{0}$ is the Bessel function of the first kind) and $\epsilon_{0}=0$. The upper band has the same quasi-energies, but increased by a constant amount of +2 , and similarly the quasi-energies of the the lower band are decreased by 2. Plotting these quantities in Fig. 4(a) demonstrates that the agreement of the perturbative result with the exact results obtained from the diagonalization of $U(T, 0)$ is extremely good, and corroborates our observation of CDT at $E=19.24$, as at this point $E / \omega=2.40$ which is the first zero of $J_{0}$. This dependence of CDT on $J_{0}$ is familiar from the behavior of the driven two-level system, ${ }^{3,17}$ in which the phenomenon of CDT was first noted.

It is interesting to observe that the quasi-energy spectrum resembles that of noninteracting electrons driven by an acfield in a superlattice, ${ }^{16,18}$ with the quasi-energy crossings corresponding to "miniband collapse." Indeed the lattice model we study can be considered to be a four-site chain with periodic boundary conditions in space. The difference between the cases arises, however, because in the case of a superlattice all intersite tunneling processes are suppressed, as the electric field is always parallel to the axis of the superlattice. Although our system is topologically equivalent to a chain, the fact that it has a two-dimensional geometry means that only tunneling processes parallel to the field are suppressed $(\mathrm{A} \leftrightarrow \mathrm{B}, \mathrm{C} \leftrightarrow \mathrm{D})$, while tunneling in directions perpendicular to the field ( $A \leftrightarrow D, B \leftrightarrow C$ ) is unaffected.

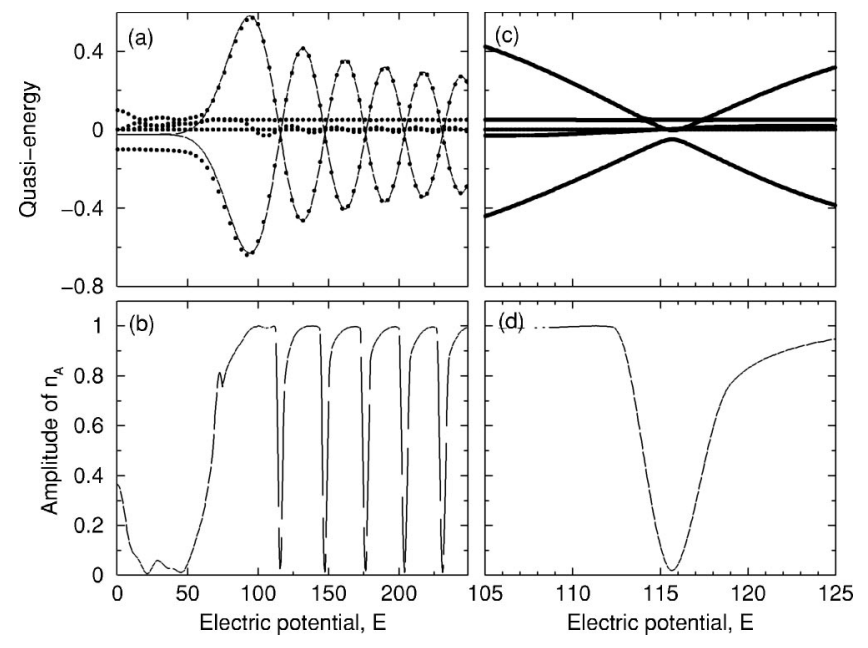

FIG. 5. (a) Quasi-energies of the system for $\widetilde{U}$ infinite, $\widetilde{V}=80$ and $\omega=8$ : circles=exact results, lines=perturbative solution $\left[ \pm 2 J_{10}(E / \omega)\right]$. (b) Amplitude of oscillation of the occupation of site A. (c) Detail of quasi-energy spectrum, showing an anticrossing. (d) Detail of amplitude of oscillations.

\section{B. Interacting electrons, no double-occupancy}

We now consider the effect of introducing interactions between the electrons, and begin by taking the Hubbard $U$-term to be infinitely large - that is, we work in the subspace of states with no double occupation. Our Hilbert space is thus six-dimensional, and we use as the basis the states shown in Fig. 2.

We show the quasi-energy spectrum of this system, again for a frequency of $\omega=8$ in Fig. 5(a). In contrast to the noninteracting case, we see that the system presents two different regimes of behavior. The first of these is the weak-field regime, $E<\tilde{V}$, at which the driving field does not dominate the dynamics. In this regime the quasi-energy spectrum, and correspondingly the amplitude of oscillations, shows little structure, and it is difficult to obtain analytical results as the perturbational approach is not valid when the tunneling terms are comparable in magnitude to the electric field.

The second regime occurs at strong field strengths, $E$ $>\widetilde{V}$, for which the quasi-energy spectrum clearly shows a sequence of close approaches. In Fig. 5(c) we show an enlargement of one of these close approaches which reveals it to be an anti-crossing. Employing the perturbation theory demonstrates that the two quasi-energies involved in these anti-crossings are described by $\pm 2 J_{n}(E / \omega)$, where $n$ is equal to $\tilde{V} / \omega$. We may thus think of $n$ as signifying the number of photons the system needs to absorb to overcome the Coulomb repulsion between the electrons occupying neighboring sites. To examine whether these anti-crossings correspond to CDT, we follow the same procedure as before, and study the amplitude of oscillations in $n_{A}$ as a function of $E$, when the system is initialized in state (6). The results in Figs. 5(b) and 5(d) strongly confirm that tunneling is highly suppressed at the anti-crossings, and hence that CDT indeed occurs.

We further show in Fig. 6 the time-dependent number occupation of the four sites at two values of $E$. In Fig. 6(a) $E$ 

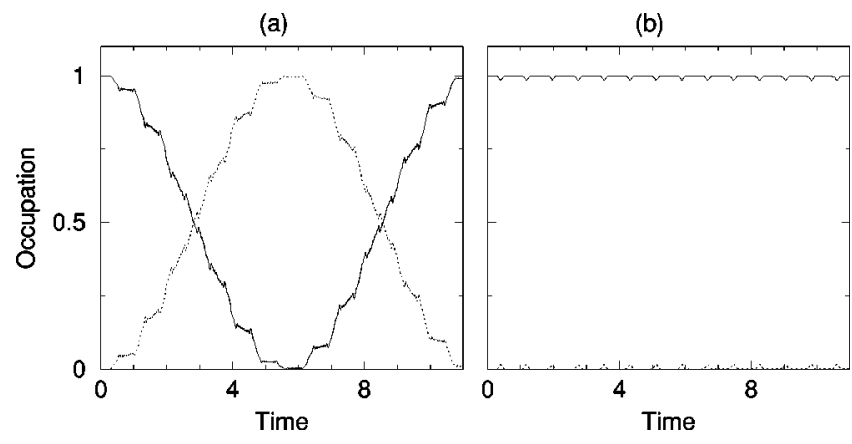

FIG. 6. Time development of the system for $\widetilde{U}$ infinite, $\widetilde{V}=80$ and $\omega=8$ : (a) electric potential, $E=100.0$ (b) $E=115.7$. Solid line indicates the occupation of sites $\mathrm{A}$ and $\mathrm{D}$, the dotted line the occupation of sites B and C.

has a value of 100.0, and it can be clearly seen that the electrons perform driven spatial Rabi oscillations between the left side of the QD and the right. Accordingly the occupation of the sites varies continuously between zero and one. In Fig. 6(b) we show the result of changing the electric potential to a value of $E=115.7$, which corresponds to the center of the first anti-crossing. In dramatic contrast to the previous case, we see that the occupation of sites A and D only varies slightly from unity, while sites $\mathrm{B}$ and $\mathrm{C}$ remain essentially empty throughout the time-evolution. Only a small amount of charge can transfer per period of the driving field between the left and right sides of the system, producing the small spikes visible in this figure. The amplitude of these features is extremely small, however, indicating that the tunneling between left and right sides has been almost totally quenched. The efficiency of the quenching depends on the value of $\widetilde{V}$ which for the case we consider is relatively high. For smaller values of $\widetilde{V}$, qualitatively the same features occur, but the efficiency of the quenching is diminished, and the sharpness of the anti-crossings is reduced.

\section{Interacting electrons, double-occupancy permitted}

We now take the most general case, and consider the competition between the $\widetilde{U}$ and $\widetilde{V}$ terms. Setting $\widetilde{U}$ to a finite value means that the four doubly-occupied basis states are no longer energetically excluded from the dynamics, and accordingly we must take the full ten-dimensional basis set.

Although it is difficult to obtain precise estimates for the values of parameters of the effective Hamiltonian, it is clear that in general $\widetilde{U}>\widetilde{V}$. Accordingly we choose the parameters $\widetilde{U}=160, \widetilde{V}=16$ to separate the two energy-scales widely for our investigation. As before we set the frequency of the acfield to $\omega=8$, and in Fig. 7(a) we show the quasi-energy spectrum obtained by sweeping over the field strength. It is immediately clear from this figure that for electric potentials $E<\widetilde{U}$ the form of the spectrum is extremely similar to the infinite- $\widetilde{U}$ case. Performing perturbation theory confirms that, as in the previous case, the behavior of the quasienergies is given by $\pm 2 J_{n}(E / \omega)$ where $n=\tilde{V} / \omega$. We show in Fig. 7(b) the amplitude of the oscillations of $n_{A}$ when the

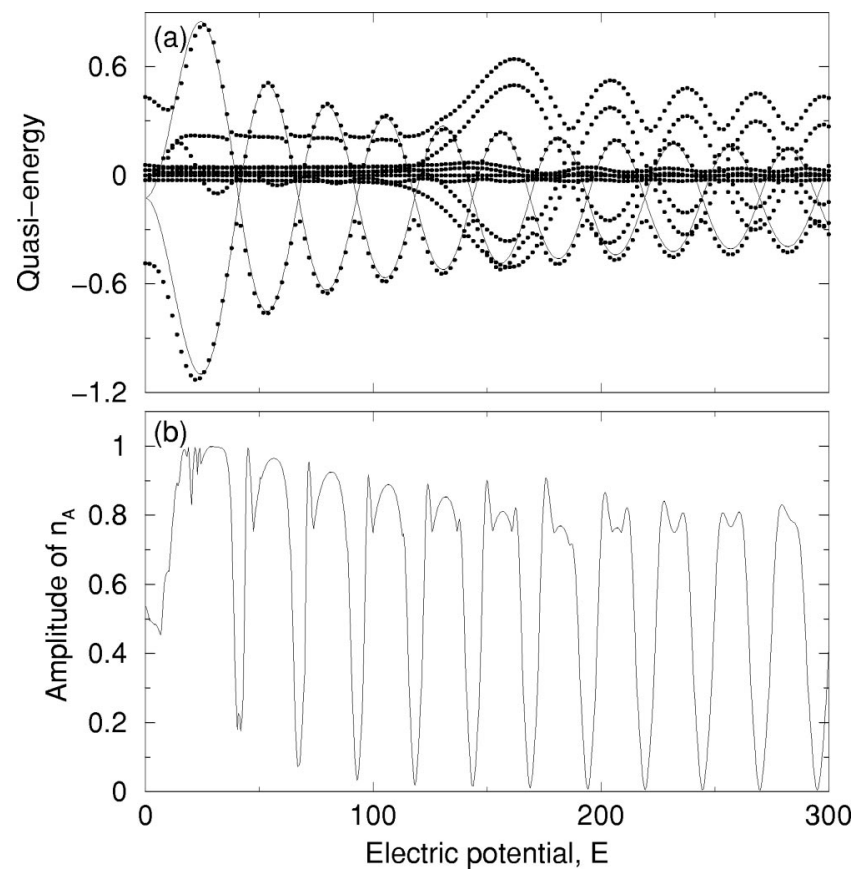

FIG. 7. (a) Quasi-energies of the system for $\widetilde{U}=160$ and $\widetilde{V}$ $=16, \omega=8$ : circles $=$ exact results, lines $=$ perturbative solution $\left[ \pm 2 J_{2}(E / \omega)\right]$. (b) Amplitude of oscillation of the occupation of site A, with (6) as the initial state.

system is initialized in state (6), which demonstrates that at the locations of the anti-crossings the tunneling parallel to the field is again quenched.

When the electric potential exceeds the value of $\widetilde{U}$, however, new structure appears in the quasi-energy spectrum. A group of four quasi-energies, that for weaker fields cluster around zero, become "excited" and make a sequence of anticrossings as the field strength is increased. The amplitude of these oscillations is comparable to the amplitude of the two quasi-energies discussed above, but it is clear that the two sets of anti-crossings are not in phase with each other. Perturbation theory predicts that these high-field quasi-energies are given by $\pm 2 J_{m}(E / \omega)$, where $m=(\widetilde{U}-\widetilde{V}) / \omega$, and thus these anti-crossings arise when the absorption of $m$ photons equates to the electrostatic energy difference between the two electrons being on neighboring sites, and doublyoccupying one site. This then indicates that this structure arises from the coupling of the ac-field to the doublyoccupied states.

To probe this phenomenon, we time-evolve the system from an initial state consisting of two electrons occupying site A. In Fig. 8(b) it can be seen that for electric potentials weaker than $\widetilde{U}$ the amplitude of the oscillations in $n_{A}$ remains small, and shows little dependence on the field. As the potential exceeds $\widetilde{U}$, this picture changes, and the ac-field drives large oscillations in $n_{A}$, and in fact mainly forces charge to oscillate between sites A and B. At the high-field anti-crossings, however, the tunneling between A and B is suppressed, which shuts down this process. Instead, the only time-evolution that the system can perform consists of undriven Rabi oscillations between sites $\mathrm{A}$ and $\mathrm{D}$, perpendicu- 

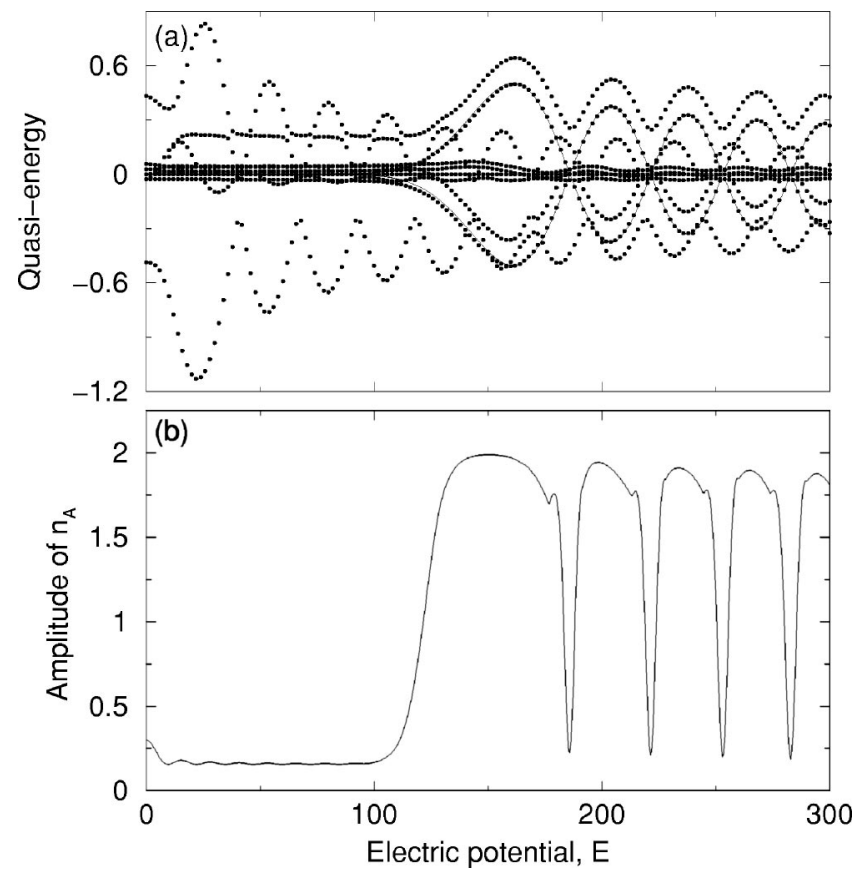

FIG. 8. (a) Quasi-energies of the system for $\widetilde{U}=160$ and $\widetilde{V}$ $=16, \omega=8$ : circles $=$ exact results, lines $=$ perturbative solution $\left[ \pm 2 J_{18}(E / \omega)\right]$. (b) Amplitude of oscillation of the occupation of site A, with site A doubly-occupied as the initial state.

lar to the field. As these oscillations are undriven they have a much longer time-scale than the forced dynamics, and thus during the interval over which we evolve the system (approximately 50 periods of the driving field), the occupation of A only changes by a small amount, producing the very sharp minima visible in Fig. 8(b), centered on the roots of $J_{m}(E / \omega)$.

As the tunneling perpendicular to the field is undriven, it is straightforward to evaluate the time evolution of the initial state, if we assume that the left side of the QD is completely decoupled from the right side. The occupation of sites $\mathrm{A}$ and $\mathrm{D}$ is then given by

$$
n_{A}(t)=1+\cos \Omega_{R} t, \quad n_{D}=1-\cos \Omega_{R} t,
$$

where $\Omega_{R}=4 \widetilde{t}^{2} /(\widetilde{U}-\widetilde{V})$, which, for the parameters we use, gives a Rabi period of $T_{R}=2 \pi / \Omega_{R}=226.19$. In Fig. 9 we display the occupations of sites $\mathrm{A}$ and $\mathrm{D}$ as a function of time, for two values of electric potential. At the first value, $E=200$, tunneling between the left and right sides of the QD is not quenched, and accordingly the occupation of the two sites varies rapidly between zero and two as the electrons are driven by the ac-field around the system. The second value, $E=185.8$, corresponds to the first high-field anti-crossing. It can be clearly seen that the charge oscillates between sites A and D, with a frequency close to $\Omega_{R}$. These Rabi oscillations are damped, however, indicating that the isolation between the left and right sides of the QD is not perfect. In this sense we can regard the two sites $\mathrm{B}$ and $\mathrm{C}$ as providing an environment, causing the quantum system composed of sites $\mathrm{A}$ and $\mathrm{D}$ to slowly decohere in time. When the tunneling between the left and right sides of the QD is strong, for ex-
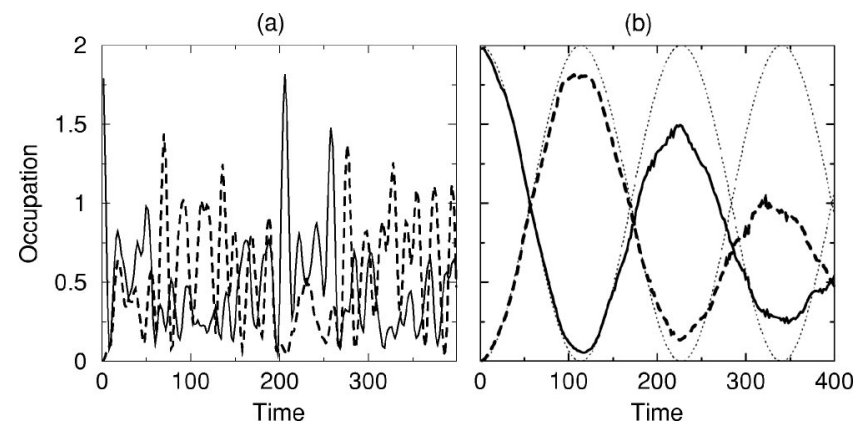

FIG. 9. Time development of the $\widetilde{U}=160$ and $\widetilde{V}=16$ system for $\omega=8$ : (a) electric potential, $\mathrm{E}=200.0$ (off-resonance) and (b) $E$ $=185.8$ (on-resonance). Thick solid line indicates the occupation of site A, the thick dotted line the occupation of site D. Dotted lines in (b) show the Rabi oscillations of the isolated two-site system, Eq. (5).

ample at $E=200$, this decoherence occurs very rapidly. By moving to an anti-crossing, however, and suppressing the tunneling, the rate of mixing between the two sides of the QD can be considerably reduced, and is just limited by the separation in energy between the two quasi-energies. Tuning the parameters of the driving field therefore gives us a simple and controllable way to investigate how a two-electron wavefunction can decohere in a QD.

\section{CONCLUSIONS}

In this article we have studied the time-dependent behavior of a system of two electrons confined to a square QD, driven by an external ac-field. By considering the strongly correlated limit of the system, we are able to use an effective lattice model of just four sites, which arises from the natural discretization presented by the system in a Wigner molecule state. We emphasize, however, that the form of the Hamiltonian we use is very general, and our results are thus applicable to a wide variety of mesoscopic systems. In our effective model, the interelectron Coulomb interaction is described by two parameters, $\widetilde{U}$ and $\widetilde{V}$, and the dynamics of the system consists essentially of tunneling from corner to corner, along the perimeter of the QD. We find that when the frequency of the driving field is in resonance with $\widetilde{V}$ or $(\widetilde{U}$ $-\widetilde{V}$ ), charge moves freely around the system, except at sharply defined field strengths at which tunneling parallel to the field is destroyed-CDT. By using Floquet theory we have established that these points correspond to the roots of $J_{m}(E / \omega)$, where $m$ is the order of the resonance [i.e., $m \omega$ $=\widetilde{V}$ or $m \omega=(\widetilde{U}-\widetilde{V})]$.

When CDT occurs, tunneling parallel to the electric field is suppressed, and the system is restricted to performing undriven Rabi oscillations in the perpendicular direction. We can therefore expect a radical modification in the frequency and polarization of the EM radiation emitted by the QD in this situation. In experiment the phenomenon of CDT should thus be readily measurable, allowing the measurement of the $\widetilde{U}$ and $\widetilde{V}$ parameters, and hence giving a simple and useful parametrization of the QD system. The tunability of this ef- 
fect, and its ability to discriminate between states of doubleoccupation and states in the single-occupation subspace, make it an excellent candidate as a control parameter for manipulating the dynamics of strongly correlated electrons in mesoscopic systems.

\section{ACKNOWLEDGMENTS}

The authors would like to thank John Jefferson for helpful discussions and comments. This research was supported by the EU through the TMR program "Quantum Transport in the Frequency and Time Domains," and by the DGES (Spain) through Grant No. PB96-0875.

\section{APPENDIX}

The full spin-dependent wavefunction of a two-electron system can be factorized into a spinor and a purely spatial wavefunction:

$$
\Psi\left(r_{1}, \sigma_{1} ; r_{2}, \sigma_{2}\right)=\chi\left(\sigma_{1}, \sigma_{2}\right) \psi\left(r_{1}, r_{2}\right),
$$

where for states of singlet symmetry the spatial wavefunction is symmetric under particle exchange (and the spinor $\chi$ is antisymmetric), while the reverse is true for triplet states. In this work we consider just the singlet sector of the model, and the two-particle states shown schematically in Fig. 2 represent the spatial components of the two-electron wavefunctions. These are formed from symmetric combinations of single-particle states defined on the lattice-points A,B,C,D, so that, for example,

$$
|1\rangle=\frac{\psi_{A}\left(r_{1}\right) \psi_{C}\left(r_{2}\right)+\psi_{C}\left(r_{1}\right) \psi_{A}\left(r_{2}\right)}{\sqrt{2}},
$$

with similar expressions holding for each of the states shown. An analogous set of states with triplet symmetry can be constructed by taking antisymmetric combinations. To complete the singlet basis we must include the four states in which a lattice point is doubly occupied, holding one spin-up electron and one spin-down, which we label as

$$
\begin{gathered}
|7\rangle=\psi_{A}\left(r_{1}\right) \psi_{A}\left(r_{2}\right), \quad|8\rangle=\psi_{B}\left(r_{1}\right) \psi_{B}\left(r_{2}\right), \\
|9\rangle=\psi_{C}\left(r_{1}\right) \psi_{C}\left(r_{2}\right), \quad|10\rangle=\psi_{D}\left(r_{1}\right) \psi_{D}\left(r_{2}\right) .
\end{gathered}
$$

Our starting point to obtain approximate expressions for the quasi-energies is Eq. (3). As the Floquet states are periodic functions of time, it is useful to work in a Hilbert space of $T$-periodic functions, ${ }^{19}$ by defining an appropriate scalar product function:

$$
\left\langle\left\langle\phi_{a}(t) \mid \phi_{b}(t)\right\rangle\right\rangle=\frac{1}{T} \int_{0}^{T}\left\langle\phi_{a}\left(t^{\prime}\right) \mid \phi_{b}\left(t^{\prime}\right)\right\rangle d t^{\prime},
$$

where the single-bracket denotes the usual inner product for the spatial component of the wavefunctions. The advantage of working in this extended Hilbert space is that the Floquet states are stationary states of the operator $\mathcal{H}(t)=H(t)$ $-i(\partial / \partial t)$, and thus we are able to employ standard stationary perturbation theory techniques, and avoid the complication of using time-dependent methods.

We first divide the Hamiltonian (1) into two parts: $H_{t}$ which contains all the tunneling terms, and $H_{I}$ containing all the interaction terms (those involving $\widetilde{U}, \widetilde{V}$ and the electric field). We then find the eigensystem of the operator $\mathcal{H}_{I}(t)$ $=H_{I}-i(\partial / \partial t)$, and employ the tunneling Hamiltonian as the perturbation. By making this division, we can expect the perturbative result to be good when tunneling is small in comparison to the other energy scales of the problem, and conversely, to break down in the limit of weak fields.

In the basis we have chosen $H_{I}$ is diagonal, with entries

$$
\begin{gathered}
H_{I}=\operatorname{diag}(0,0, \widetilde{V}, \widetilde{V}-E(t), \widetilde{V}, \widetilde{V}+E(t), \widetilde{U}+E(t), \\
\widetilde{U}-E(t), \widetilde{U}-E(t), \widetilde{U}+E(t)),
\end{gathered}
$$

where $E(t)=E \cos \omega t$, and thus finding the eigensystem of $\mathcal{H}_{I}(t)$ reduces to the straightforward task of solving the ten first-order differential equations:

$$
\left[\left(H_{I}\right)_{j j}-i \frac{d}{d t}\right] \phi_{j}(t)=\epsilon_{j} \phi_{j}(t) .
$$

For $j=1$ and 2, (A5) has the trivial solutions $\phi_{j}(t)$ $=1, \epsilon_{j}=0$. The third and fifth components also do not have an explicit time dependence, and so have similarly simple solutions: $\phi_{j}(t)=\exp \left[i\left(\epsilon_{j}-\widetilde{V}\right) t\right]$. Imposing periodic boundary conditions sets the value of the quasi-energy, requiring $\left(\epsilon_{j}\right.$ $-\widetilde{V})=m \omega$ where $m$ is an integer. The remaining solutions are all similar in form to each other, and as an example, $\phi_{10}(t)$ is given by

$$
\phi_{10}(t)=\exp \left[-i\left(\widetilde{U}-\epsilon_{10}\right) t-i \frac{E}{\omega} \sin \omega t\right] .
$$

Imposing periodic boundary conditions on the this solution requires $(\widetilde{U}-\epsilon)=n \omega$, where $n$ is an integer.

The eigenvalues of $\mathcal{H}_{I}$ are thus 0 (with a twofold degeneracy), $\widetilde{V} \bmod \omega$ (with a fourfold degeneracy), and $\widetilde{U} \bmod \omega$ (also with a fourfold degeneracy). These represent the zeroth-order approximations to the quasi-energies in the perturbational expansion. It can readily be shown that their associated eigenvectors $\phi_{j}(t)$ form an orthonormal basis set, and, by using standard degenerate perturbation theory, the quasi-energies can be obtained to first-order by finding the eigenvalues of the perturbing operator $\left\langle\left\langle\phi_{i}\left|H_{t}\right| \phi_{j}\right\rangle\right\rangle$. By using the identity

$$
\exp [-i \beta \sin \omega t]=\sum_{m=-\infty}^{\infty} J_{m}(\beta) \exp [-i m \omega t]
$$

to rewrite the eigenvectors which have the form (A6), the 
scalar products (A3) can be evaluated straightforwardly, allowing the matrix elements of the perturbing operator to be obtained, and the operator to be subsequently diagonalized.

For the general case, with $\widetilde{V}=m \omega$ and $\widetilde{U}=n \omega$, the firstorder approximation to the quasi-energies can be readily shown to be 0 (with a fourfold degeneracy), $\pm 2 J_{n}(E / \omega)$, and $\pm 2 J_{n-m}(E / \omega)$ (with each state being twofold degenerate). The specific cases treated in Secs. III A and B can be treated in a similar way, giving the perturbative solutions quoted there.
${ }^{1}$ T.H. Oosterkamp, T. Fujisawa, W.G. van der Wiel, K. Ishibashi, R.V. Hijman, S. Tarucha, and L.P. Kouwenhoven, Nature (London) 395, 873 (1998).

${ }^{2}$ B.E. Cole, J.B. Williams, B.T. King, M.S. Sherwin, and C.R. Stanley, Nature (London) 410, 60 (2001); D. Vion, A. Aasime, A. Cottet, P. Joyez, H. Pothier, C. Urbina, D. Esteve, and M.H. Devoret, Science 296, 886 (2002).

${ }^{3}$ F. Grossmann, T. Dittrich, P. Jung, and P. Hänggi, Phys. Rev. Lett. 67, 516 (1991).

${ }^{4}$ M. Grifoni and P. Hänggi, Phys. Rep. 304, 229 (1998).

${ }^{5}$ J.H. Jefferson and W. Häusler, Phys. Rev. B 54, 4936 (1996).

${ }^{6}$ S. Akbar and I.-H. Lee, Phys. Rev. B 63, 165301 (2001).

${ }^{7}$ C.E. Creffield, W. Häusler, J.H. Jefferson, and S. Sarkar, Phys. Rev. B 59, 10719 (1999).

${ }^{8}$ C.E. Creffield, J.H. Jefferson, S. Sarkar, and D.L.J. Tipton, Phys. Rev. B 62, 7249 (2000).

${ }^{9}$ M. Koskinen, M. Manninen, B. Mottelson, and S.M. Reimann,
Phys. Rev. B 63, 205323 (2001).

${ }^{10}$ D.G. Austing, T. Honda, and S. Tarucha, Semicond. Sci. Technol. 12, 631 (1997).

${ }^{11}$ J.T. Stockburger, Phys. Rev. E 59, R4709 (1999).

${ }^{12}$ C.A. Stafford and S. Das Sarma, Phys. Rev. Lett. 72, 3590 (1994); R. Kotlyar and S. Das Sarma, Phys. Rev. B 55, R10205 (1997).

${ }^{13}$ C.S. Lent, Science 288, 159 (2000); G.H. Bernstein, I. Amlani, A.O. Orlov, C.S. Lent, and G.L. Snider, Nanotechnology 10, 166 (1999).

${ }^{14}$ C.E. Creffield and G. Platero, Phys. Rev. B 65, 113304 (2002).

${ }^{15}$ T. Fujisawa, D.G. Austing, Y. Tokura, Y. Hirayama, and S. Tarucha, Phys. Rev. Lett. 88, 236802 (2002).

${ }^{16}$ M. Holthaus, Z. Phys. B: Condens. Matter 89, 251 (1992).

${ }^{17}$ J.H. Shirley, Phys. Rev. 138, B979 (1965).

${ }^{18}$ M. Holthaus, Phys. Rev. Lett. 69, 351 (1992).

${ }^{19}$ H. Sambe, Phys. Rev. A 7, 2203 (1973). 